\title{
Adipose Tissue Dysfunction in Nascent Metabolic Syndrome
}

\author{
Andrew A. Bremer ${ }^{1}$ and Ishwarlal Jialal ${ }^{2,3}$ \\ ${ }^{1}$ Department of Pediatrics, Vanderbilt University, Nashville, TN 37232-9170, USA \\ ${ }^{2}$ Laboratory for Atherosclerosis and Metabolic Research, University of California Davis Medical Center, \\ Sacramento, CA 95817-2218, USA \\ ${ }^{3}$ VA Medical Center, Mather, CA 95655-4200, USA \\ Correspondence should be addressed to Ishwarlal Jialal; ijialal@ucdavis.edu
}

Received 26 January 2013; Accepted 14 February 2013

Academic Editor: Anne E. Sumner

Copyright ( 2013 A. A. Bremer and I. Jialal. This is an open access article distributed under the Creative Commons Attribution License, which permits unrestricted use, distribution, and reproduction in any medium, provided the original work is properly cited.

\begin{abstract}
The metabolic syndrome (MetS) confers an increased risk for both type 2 diabetes mellitus (T2DM) and cardiovascular disease (CVD). Moreover, studies on adipose tissue biology in nascent MetS uncomplicated by T2DM and/or CVD are scanty. Recently, we demonstrated that adipose tissue dysregulation and aberrant adipokine secretion contribute towards the syndrome's low-grade chronic proinflammatory state and insulin resistance. Specifically, we have made the novel observation that subcutaneous adipose tissue (SAT) in subjects with nascent MetS has increased macrophage recruitment with cardinal crown-like structures. We have also shown that subjects with nascent MetS have increased the levels of SAT-secreted adipokines (IL-1, IL-6, IL-8, leptin, RBP-4, CRP, SAA, PAI-1, MCP-1, and chemerin) and plasma adipokines (IL-1, IL-6, leptin, RBP-4, CRP, SAA, and chemerin), as well as decreased levels of plasma adiponectin and both plasma and SAT omentin-1. The majority of these abnormalities persisted following correction for increased adiposity. Our data, as well as data from other investigators, thus, highlight the importance of subcutaneous adipose tissue dysfunction in subjects with MetS and its contribution to the proinflammatory state and insulin resistance. This adipokine profile may contribute to increased insulin resistance and low-grade inflammation, promoting the increased risk of T2DM and CVD.
\end{abstract}

\section{Introduction}

The metabolic syndrome (MetS) comprises a cluster of cardiometabolic risk markers with insulin resistance and adiposity as central features [1-4]. Five diagnostic criteria for MetS have been identified (central obesity, dyslipidemia (high triglycerides (TGs) and/or low high-density lipoprotein cholesterol (HDL-C)), hypertension, and impaired fasting glucose) by the Adult Treatment Panel III (ATPIII) criteria of the National Cholesterol and Education Program (NCEP), and the presence of three of these features is considered sufficient to diagnose the syndrome $[2,4,5]$. Using this definition, the National Health and Nutrition Examination Survey (NHANES) data show that currently $~ 35 \%$ of all US adults have MetS [6] and that $>40 \%$ of adults over the age of 50 have the syndrome [7]. It is important to emphasize that the diagnosis of MetS has been harmonized using the NCEP ATPIII criteria with the exception of different cut-points for waist circumference for different races [8]. Furthermore, MetS confers an increased risk for cardiovascular disease (CVD) and type 2 diabetes mellitus (T2DM) [7, 9-12], both of which are additional risk factors for increased morbidity and mortality.

Numerous investigators have shown increased circulating biomarkers of inflammation in MetS, thus providing support for the syndrome's proinflammatory state [2, 4, 13]. Furthermore adipokine biology has been extensively detailed in recent reviews, and hence it will not be the focus of this paper [14-16]. However, there are scant data on adipose tissue biology in individuals with nascent MetS (a term coined by us to denote subjects with MetS but without the confounding presence of diabetes and/or cardiovascular diseases) [17]. The relationship between inflammation and MetS is supported by several studies $[2,4,18,19]$, as is the relationship between increased visceral fat mass and MetS [20-22]. However, there is a paucity of data on subcutaneous adipose tissue (SAT) biology in the pathogenesis of MetS [23]. 
The subcutaneous fat-which comprises $\sim 80 \%$ of adipose tissue and is the major source of fatty acids for the liver-is readily accessible to study and has been shown to be metabolically correlated to indices of insulin resistance as well as to visceral adipose tissue (VAT) [24-27]. In addition to intraabdominal fat, investigators have shown that the amount of SAT in subjects with MetS positively correlates with increasing MetS factor scores and negatively correlates with circulating adiponectin levels [28]. Other investigators have also reported that SAT is significantly associated with MetS and increases with the increasing number of MetS features, independent of age and sex [29]. Furthermore, inflammatory cells and processes, such as macrophage infiltration, appear to be important in adipose tissue inflammation. Specifically, investigators have examined abdominal SAT from obese subjects and reported that an inflamed adipose phenotype characterized by tissue macrophage accumulation in crownlike structures (CLSs) is associated with systemic hyperinsulinemia and insulin resistance and impaired endotheliumdependent flow-mediated vasodilation [30]. Macrophage retention in fat was also linked to upregulated tissue CD68 and tumor necrosis factor-alpha (TNF- $\alpha$ ) mRNA expressions in addition to increased plasma high-sensitivity C-reactive protein (hsCRP) concentrations.

Although it appears that chronic low-grade inflammation could be a central feature to explain the increased risks of CVD and diabetes in MetS, the precise mechanisms remain to be elucidated. As such, our laboratory has focused on the potential role of SAT dysregulation in the syndrome's pathogenesis. Specifically, given the paucity of data examining SAT biology and plasma adipokines in subjects with nascent MetS, we have investigated the role of SAT in MetS and its contribution to the syndrome's systemic low-grade inflammatory process.

\section{Subcutaneous Adipose Tissue Dysregulation in Nascent MetS}

To determine whether SAT biology in subjects with nascent MetS is dysregulated and contributes to the syndrome's systemic low-grade inflammatory process, we studied 65 ageand sex-matched adults [31]. Subjects were classified as having MetS or not using the NCEP ATPIII criteria [5]; those classified as MetS had at least three risk factors to sustain the diagnosis, including central obesity, hypertension, dyslipidemia (low HDL-C and high TGs), impaired fasting glucose, and/or hypertension or on antihypertensive medications. The control subjects needed to have $\leq 2$ features of MetS and not be on blood pressure (BP) medications. Other exclusion criteria for controls were a fasting plasma glucose concentration $>100 \mathrm{mg} / \mathrm{dL}$ and a fasting TG concentration $>200 \mathrm{mg} / \mathrm{dL}$. For both groups, other exclusion criteria were a previous diagnosis of diabetes, clinical atherosclerosis (coronary artery disease (CAD), peripheral vascular disease, CVD, etc.), a TG concentration $>400 \mathrm{mg} / \mathrm{dL}$, a hsCRP concentration $>10 \mathrm{mg} / \mathrm{L}$, pregnancy, an increased complete blood count (CBC), alcohol consumption $>1$ oz/day, consumption of $n-3$ polyunsaturated fatty acids, smoking, hypo- or hyperthyroidism, malabsorption, active wounds, recent surgery, inflammatory or malignant disease, anticoagulant therapy, steroid therapy, the current use of anti-inflammatory drugs, statins and/or other hypolipidemic agents, hypoglycemic agents, angiotensin receptor blockers, oral contraceptives, and antioxidant supplements (in the prior 6 months), postmenopausal women on estrogen replacement therapy, and chronic high intensity exercisers (exercise $>100 \mathrm{~min} /$ week).

Fasting plasma samples were collected from all volunteers after informed consent, and SAT biopsies were obtained from the gluteal area. Biomarkers that were examined included adiponectin, CRP, serum amyloid A (SAA), leptin, plasminogen activator inhibitor-1 (PAI-1), retinol-binding protein4 (RBP-4), chemokines (monocyte chemotactic protein-1 (MCP-1), and interleukin (IL)-8), as well as cytokines (IL1, TNF- $\alpha$, IL-8, and IL-6). In addition, SAT samples were stained for CD68 (a macrophage marker) and T-cells (CD3 and CD5) to assess macrophage/T-cell infiltration into the adipose tissue, and the numbers of CLS per high power field (HPF) were counted. There were no significant differences in the ages of the participants and the male: female ratio between the controls and subjects with MetS. Since the percent of African Americans in our cohort was only 9 percent [32], we were unable to undertake any realistic subgroup analyses with respect to race.

Not surprisingly, the waist circumference (WC), body mass index (BMI), BP, fasting glucose concentrations, nonHDL-cholesterol concentrations, TG concentrations, and the homeostasis model assessment (HOMA) for insulin resistance were higher in the MetS subjects than in controls, whereas HDL-C concentrations were lower. Furthermore, the hsCRP, IL-6, IL-1 $\beta$, leptin, SAA, and RBP- 4 concentrations were significantly higher in the MetS subjects than in controls, whereas the adiponectin concentrations were lower.

Table 1 shows the concentrations of several biomarkers released from incubated SAT specimens from the controls and subjects with MetS. Expressed per gram of fat, the levels of secreted leptin, RBP-4, CRP, SAA, PAI-1, and MCP-1 were significantly higher in subjects with MetS than controls. Moreover, the SAT release of IL- $1 \beta$, IL-6, IL-8, and MCP-1, as expressed per mg of protein, was higher in SAT from subjects with MetS than controls.

No lymphocyte populations were observed in any of the SAT specimens from the controls and subjects with MetS using CD3 and CD5 staining. However, there were significantly increased numbers of macrophages infiltrating the SAT of MetS subjects compared to controls as demonstrated by positive CD68 staining. Furthermore, there were significantly increased numbers ( 3-fold) of CLS in the SAT specimens from MetS subjects than those from controls (controls: 5 CLS/10 hpf; MetS: 14 CLS/10 hpf; $P<0.001$ ). Interestingly, the CLS did not correlate with any proinflammatory mediators, suggesting that they are not classical M1 macrophages [31]. The elucidation of the SAT macrophage phenotype in subjects with MetS is critical to understanding its role in the syndrome's pathogenesis.

Since the patients with MetS in our study cohort had significantly greater WCs than the controls, all the analytes were also evaluated with WC as a covariate. Importantly, in the adjusted analyses, all the reported differences between 
TABLE 1: Subcutaneous adipose tissue biomarker levels.

\begin{tabular}{lcc}
\hline & Controls & MetS \\
\hline Adiponectin (ng/g) & $4.2(1.3,5.6)$ & $3.7(1.2,4.6)$ \\
Leptin (ng/g) & $3.0(2.1,6.2)$ & $7.3(3.8,18.6)^{*}$ \\
RBP-4 (ng/g) & $11.1(6.4,18.4)$ & $29.1(16.2,33.7)^{* *}$ \\
CRP (ng/g) & $2.5(2.3,7.9)$ & $5.4(3.4,19.1)^{*}$ \\
SAA (ng/g) & $14.8(5.1,34.2)$ & $25.3(14.5,55.7)^{*}$ \\
PAI-1 (ng/g) & $3.2(2.2,6.5)$ & $5.6(3.1,9.9)^{* *}$ \\
MCP-1 (ng/g) & $6.7(4.3,9.1)$ & $22.1(11.8,33.5)^{* *}$ \\
IL-1 $\beta$ (ng/mg protein) & $31.1(21.2,45.1)$ & $39.7(24.8,61.5)^{*}$ \\
TNF (ng/mg protein) & $3.7(1.9,4.6)$ & $3.8(2.9,5.3)$ \\
IL-6 (ng/mg protein) & $16.5(10.6,24.5)$ & $18.7(12.7,33.2)^{*}$ \\
IL-8 (ng/mg protein) & $10.9(5.1,14.2)$ & $17.4(14.5,27.3)^{* *}$ \\
\hline
\end{tabular}

Copyright 2011, The Endocrine Society. Reproduced with permission from the Endocrine Society.

Data are expressed as median (25th percentile and 75th percentile).

${ }^{*} P<0.05,{ }^{* *} P<0.001$ compared to controls.

the MetS and control groups persisted except for RBP-4 (Table 1). Furthermore, a subgroup analysis revealed higher levels of only leptin and RBP-4 in those subjects with a WC over the median, $42 \mathrm{in}$. versus under $42 \mathrm{in}$. (for $\mathrm{WC}<42 \mathrm{in}$., leptin was $71(49-83) \mathrm{ng} / \mathrm{ml}$ versus 82 (55-89) $\mathrm{ng} / \mathrm{ml}$ for WC $>42$ in.; for WC $<42$ in., RBP-4 was 25 (11-31) g/mg protein versus 33 (17-37) ng/mg protein for $\mathrm{WC}>42$ in.; $P<0.05$ compared with WC $<42$ in.).

Because both insulin resistance and low-grade inflammation are typical features of MetS, we used HOMA and hsCRP as the representative biomarkers for these conditions to evaluate relevant correlations as well. HsCRP correlated positively with HOMA ( $r=0.39, P=0.03)$ and adipose tissue MCP-1 $(r=0.46, P=0.03)$ and negatively with adipose tissue adiponectin $(r=-0.44, P=0.01)$. There were significant correlations between circulating CRP and SAT CRP $(r=0.49$, $P=0.01), \mathrm{IL}-1 \beta(r=0.56, P=0.002)$, and IL-6 $(r=0.76$, $P=0.001)$ as well. Furthermore, HOMA correlated positively with PAI-1 $(r=0.56, P=0.02)$, RBP-4 $(r=0.49, P=0.03)$, and SAT MCP- $1(r=0.039, P=0.04)$.

Given the sparse data on adipose tissue biology in nascent MetS, we recently reported our findings evaluating four additional novel adipokines in this cohort, including chemerin, omentin-1, resistin, and visfatin in both plasma and SAT [17]. Importantly, as noted above, none of the subjects studied had diabetes or any chronic inflammatory diseases nor were any on anti-inflammatory, hypolipidemic, or hypoglycemic drugs. Furthermore, all the subjects had CRP levels $<10 \mathrm{mg} / \mathrm{L}$, normal CBCs, fasting glucose concentrations between 100 and $125 \mathrm{mg} / \mathrm{dL}$, and a hemoglobin Alc (HbAlc) <6.5\%. Not surprisingly, subjects with MetS were more insulin resistant and had higher levels of hsCRP. It is important to emphasize that in this paper 20 percent of the patients with MetS were not obese.

The circulating levels of chemerin were significantly increased in the subjects with MetS compared to controls, and this significance persisted $(P=0.0005)$ following adjustment for age and BMI or WC (Table 2). There were also significantly lower levels of plasma omentin-1 in subjects with MetS compared to controls $(P=0.004)$; importantly, the significance persisted when the data were adjusted for BMI or WC (Table 2) $(P=0.03)$. Furthermore, there were higher levels of circulating resistin in subjects with MetS compared to controls; however, these differences did not persist following adjustment for BMI or WC. Plasma visfatin levels were not significantly different between the two groups.

Importantly, there was also a significantly higher release of chemerin from SAT in subjects with MetS which persisted following adjustment for BMI or WC and age (Table 2). In addition, there was a significantly lower secretion of omentin from SAT in subjects with MetS which persisted following adjustment for both age and BMI or WC. However, the secretion of both resistin and visfatin from SAT was not significantly different between the MetS and control groups. Plasma chemerin concentrations correlated significantly $(P<$ $0.05)$ with SAT chemerin $(r=0.44)$, hsCRP $(r=0.28)$, HOMA $(r=0.42)$, TG $(r=0.41)$, systolic BP $(r=0.28)$, omentin $(r=-0.42)$, and HDL-C $(r=-0.37)$. Moreover, both circulating and SAT omentin-1 levels correlated significantly with each other $(r=0.44, P<0.05)$. Plasma omentin-1 concentrations also correlated significantly with glucose $(r=$ $-0.38)$, TG $(r=-0.48)$, and HDL-C $(r=0.52)$ levels.

\section{Discussion}

We have shown that SAT biomarkers and architecture differ markedly in subjects with MetS compared to matched controls [31]. Specifically, we have documented that patients with nascent MetS (without the confounding conditions of diabetes and/or CVD) have increased levels of adipokines and decreased levels of adiponectin that are related to insulin resistance and inflammation. Furthermore, we have shown that this dysregulation of adipokines is not accounted for simply by increased adiposity, suggesting that other aspects of MetS contribute to both the proinflammatory and insulin resistant states of the syndrome. Also MetS should be classified as a high-risk obesity state based on our findings. Furthermore, at least 20 percent of our cohort were not obese, underscoring the high risk of metabolic syndrome in both obese and nonobese individuals.

Furthermore, we have documented a significant increase in macrophages in SAT and abundant CLS which appear to surround a hypoxic environment triggered by adipocyte death in subjects with MetS [33-35]. For unclear reasons, there were no significant correlations between CLS and biomarkers of inflammation in our studies; however, one can speculate that the CLS could be predominantly of the M2 macrophage phenotype participating in tissue remodeling. Also, we have shown that the chemokine MCP-1 is increased in SAT from MetS subjects, and, since this chemokine facilitates the homing of macrophages to such tissue depots, our data suggest that the SAT may indeed be a key player in MetS and its associated comorbidities. Moreover, because SAT is easy to access and SAT biopsies can be performed in large-scale clinical studies, and since the expression of inflammatory genes in SAT compares well with VAT $[25,26]$, 
TABLE 2: Novel adipokine concentrations.

\begin{tabular}{|c|c|c|c|}
\hline Variable & Controls $(n=30)$ & MetS $(n=45)$ & $\begin{array}{c}P \text { value } \\
\text { MetS versus control }\end{array}$ \\
\hline hsCRP (mg/L) & $1.3(0.5,4.0)$ & $3.1(1.6,5.4)$ & 0.006 \\
\hline HOMA-IR & $1.1(1.0,2.8)$ & $2.8(1.9,5.1)$ & 0.0001 \\
\hline \multirow{2}{*}{ Plasma chemerin $(\mathrm{ng} / \mathrm{mL})$} & $271 \pm 53$ & $366 \pm 64$ & $<0.0001$ \\
\hline & $n=20$ & $n=37$ & ${ }^{*}(0.0005)$ \\
\hline \multirow{2}{*}{ SAT chemerin (ng/mg protein) } & $3.05 \pm 0.94$ & $3.94 \pm 0.74$ & \multirow{2}{*}{0.001} \\
\hline & $n=30$ & $n=45$ & \\
\hline \multirow{2}{*}{ Plasma omentin (ng/mL) } & $27 \pm 14$ & $16 \pm 5$ & 0.004 \\
\hline & $n=16$ & $n=16$ & ${ }^{*}(0.03)$ \\
\hline \multirow{2}{*}{ SAT omentin (ng/mg protein) } & $0.31 \pm 0.09$ & $0.22 \pm 0.10$ & \multirow{2}{*}{0.01} \\
\hline & $n=30$ & $n=45$ & \\
\hline \multirow{2}{*}{ Plasma resistin $(\mathrm{ng} / \mathrm{mL})$} & $1.8(1.5,2.5)$ & $2.4(1.7,3.1)$ & 0.04 \\
\hline & $n=21$ & $n=31$ & ${ }^{*}(0.07)$ \\
\hline \multirow{2}{*}{ SAT resistin (ng/mg protein) } & $0.16 \pm 0.06$ & $0.17 \pm 0.05$ & \multirow{2}{*}{ NS } \\
\hline & $n=30$ & $n=45$ & \\
\hline \multirow{2}{*}{ Plasma visfatin (ng/mL) } & $0.57(0.38,0.71)$ & $0.59(0.31,0.96)$ & \multirow{2}{*}{$\begin{array}{c}0.14 \\
*(0.13)\end{array}$} \\
\hline & $n=22$ & $n=36$ & \\
\hline \multirow{2}{*}{ SAT visfatin (ng/mg protein) } & $0.17 \pm 0.09$ & $0.21 \pm 0.1$ & \multirow{2}{*}{ NS } \\
\hline & $n=30$ & $n=45$ & \\
\hline
\end{tabular}

${ }^{*}(P$ value adjusted for age and $\mathrm{BMI})$.

Results are presented as mean \pm standard deviation or median (25th percentile and 75 th percentile).

the evaluation of SAT in subjects with and without MetS may provide novel insights into the syndrome's pathogenesis and serious sequelae.

Our data are also consistent with some but not all studies that have reported $\mathrm{mRNA}$ /gene expression profiles in SAT in subjects with MetS [36, 37]. Specifically, Gormez et al. reported increased mRNA levels of TNF- $\alpha$ and leptin but not adiponectin in SAT from subjects with MetS versus controls [36], whereas our data showed only increased amounts of SAT-secreted and circulating leptin concentrations. However, Gormez et al. studied MetS patients with CAD with $88 \%$ of the subjects having concomitant diabetes and dyslipidemia. Thus, one cannot appropriately ascribe their findings to MetS alone, and the additional confounding effects of medical conditions and medications cannot be excluded. Furthermore, Sacks et al. [37] reported no changes with respect to IL- $1 \beta$ gene expression levels in SAT from 15 subjects with MetS with CAD versus controls, whereas our data (using a much larger sample size) showed an increased amount of IL- $1 \beta$ released from SAT and in plasma. However, unlike in our studies, Sacks et al. [37] focused on a few selective biomediators/biomarkers with the confounding of CAD. The reported differences could also be the result of posttranscriptional processes, and thus highlight the problem with inferring that gene expression directly correlates with protein expression, the focus of our studies since it denotes function.

The novelty of our study is that we evaluated subjects with nascent MetS (without the confounding of diabetes and/or CVD) and assayed both plasma and SAT-secreted levels of adipokines. It is imperative to study MetS at this nascent stage in order to obtain a better understanding of the pathophysiology of this common disorder. Thus, our data suggest that these circulating adipokines function as biomediators that contribute to the increased risk for both diabetes and CVD in MetS subjects [38-40].

It is also important to note that CRP levels are significantly increased in the SAT of MetS subjects compared to controls. Although CRP appears to be predominantly produced in the liver, previous investigators have shown that there is increased CRP gene expression from adipose tissue [41] and vascular endothelium [42], a component of the stromal vascular fraction. These data thus support the notion that MetS is a proinflammatory state and that the adipose tissue of MetS contributes to the increased inflammation of these subjects.

The essential role of SAT in metabolic homeostasis has best been described in lipodystrophic syndromes, where its absence leads to ectopic fat accumulation in the liver and skeletal muscle with concomitant insulin resistance [43]. Although a deficiency in SAT has been previously associated with MetS, our data demonstrate that the SAT may also be a key player in the pathogenesis of MetS, consistent with findings from the Framingham Heart Study showing that larger volumes of SAT were associated with more cardiometabolic risk factors [22].

Another important observation from our studies is that MCP-1 appears to correlate well with insulin resistance and inflammation. Previous studies in rodents have shown that knockout of the MCP-1 receptor results in decreased hepatic 


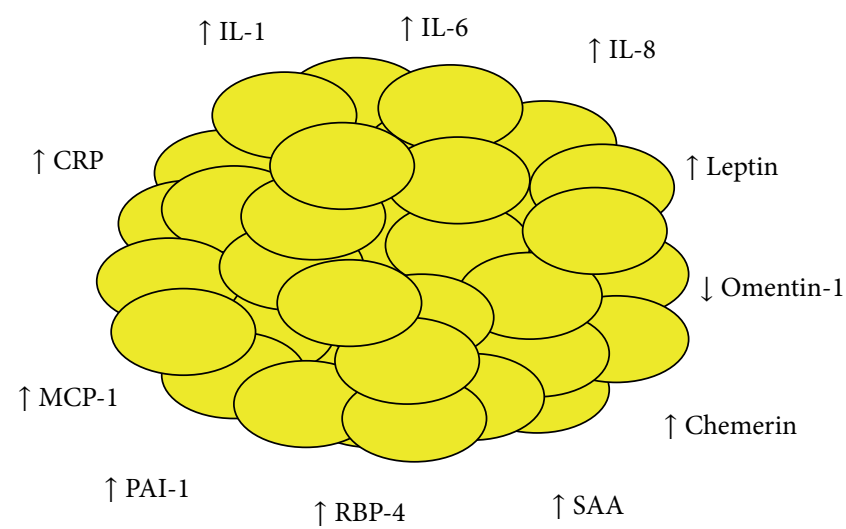

FIGURE 1: Schema depicting adipokine dysfunction in metabolic syndrome. Abbreviations: MCP-1, monocyte chemotactic protein-1; RBP-4, retinol-binding protein-4; SAA, serum amyloid A; CRP, C-reactive protein; IL, interleukin; PAI-1, plasminogen activator inhibitor-1.

inflammation and steatosis [44] and decreased adipose tissue macrophages [45], underscoring the importance of SAT MCP-1 in MetS subjects. The exact mechanisms by which MCP-1 contributes to both insulin resistance and increased inflammation in MetS need to be elucidated in future studies.

Moreover, we have made the novel observation that the SAT in subjects with nascent MetS (uncomplicated by the comorbidities of diabetes and/or CVD) has increased macrophage recruitment with cardinal CLS in greater abundance. Furthermore, these cells in SAT conspire to produce increased levels of biomarkers that correlate with both insulin resistance and low-grade inflammation, potentially presaging the subsequent increased risk for diabetes and CVD.

In addition, we have recently demonstrated abnormal circulating and SAT-secreted chemerin and omentin-1 levels in subjects with nascent MetS [17]. Chemerin is a novel adipokine that is produced by both adipose tissue and liver; moreover, it is a chemoattractant for immune cells such as macrophages and promotes adipocyte differentiation [46]. Chemerin levels have also been shown to be higher in obesity, some features of MetS, diabetes, and nonalcoholic fatty liver disease [46-48], and it appears to induce insulin resistance in skeletal muscle, the major site of peripheral insulin resistance [49]. In our studies, we have made the novel observation that both plasma and SAT levels of chemerin are higher in subjects with nascent MetS, suggesting that chemerin could be involved early in the pathogenesis of the syndrome. Previously, in Caucasian subjects with Mets (including some with concomitant diabetes), serum chemerin levels were reported to be significantly increased; however, they were not adjusted for adiposity [50]. However, the investigators did not find a correlation between insulin resistance, obtained by two measures (HOMA and the quantitative insulin sensitivity check index (QUICKI)) and chemerin concentrations [50]. In a subsequent study in Korean subjects [51], the authors suggested that the ratio of chemerin to adiponectin might be a good predictor of MetS but did not report on adiposityadjusted differences between patients with MetS and controls. Also, Dong et al. reported increased chemerin levels in patients with MetS (41\% on statin therapy) with and without
$\mathrm{CAD}$ and suggested it was an independent predictor of angiographic CAD [52]. However, they too did not correct for adiposity compared to controls, and thus we are unclear if this is a manifestation of MetS per se. Nonetheless, these studies collectively suggest a role for chemerin in the pathogenesis of MetS and its use as a biomarker to predict the syndrome needs to be urgently elucidated.

Furthermore, based on our investigations, it appears that higher plasma chemerin levels in subjects with MetS emanate largely from the adipose tissue; however, we cannot exclude the contribution of other sources of chemerin production such as the liver. But, since VAT is not a major source of chemerin [53], our studies highlight the contribution of SAT to circulating chemerin levels and its use as a potential biomarker of SAT dysregulation. Moreover, our findings demonstrate higher SAT and plasma chemerin concentrations independent of obesity in nascent MetS, and also confirm significant correlations with insulin resistance, inflammation, BP, and dyslipidemia in nascent MetS, suggesting a potential role of chemerin in MetS and its sequelae [46-49].

As opposed to chemerin, omentin is predominantly expressed and secreted by VAT $[54,55]$ and appears to have insulin-sensitizing actions [55]. Furthermore, its levels are lower with both obesity and diabetes $[55,56]$. We have documented lower levels of omentin-1 in nascent MetS in both SAT and plasma; moreover, lower omentin-1 levels persisted following correction for obesity in both plasma and in SAT [13]. Shang et al. have reported lower serum omentin1 levels in patients with MetS $(23 \%$ on statins and $32 \%$ on angiotensin-converting enzyme inhibitors/angiotensin receptor blockers) [57]; however, they did not correct for the increased BMI and waist circumference. Thus, they were unable to conclude that this correlation is a feature of MetS per se. Our study therefore adds to the published literature by documenting lower omentin release from SAT in subjects with nascent MetS independent of obesity. Moreover, since adipose tissue is the major source of omentin, we suggest that the lower secretion of omentin from SAT in subjects with nascent MetS establishes the presence of omentin deficiency in the syndrome as well. 
In our studies, omentin levels significantly correlated with glucose $(r=-0.43)$, TG $(r=-0.50)$, and HDL-C $(r=$ 0.53 ) concentrations, all features of MetS, but not with CRP levels and HOMA. Furthermore, although we showed higher plasma resistin concentrations in the subjects with MetS (that corrected with adiposity) versus the controls, we did not demonstrate higher SAT resistin levels. Thus, we can conclude that this cytokine, which in humans arises mainly from activated leukocytes [58], is a marker for the higher leuckoyte activity that we reported previously $[59,60]$. Moreover, since visfatin levels were not different in MetS versus control subjects, we cannot confirm a role for visfatin in the etiology of MetS. A schematic representation of the induced SAT dysregulation in subjects with nascent MetS is depicted in Figure 1.

\section{Conclusions}

We have made the novel observations that (i) the SAT in subjects with nascent MetS has increased macrophage recruitment with CLS in greater abundance; (ii) the SAT in subjects with nascent MetS produces increased levels of biomarkers that correlate with both insulin resistance and low-grade inflammation, potentially presaging the subsequent increased risk for diabetes and CVD; and (iii) there is a dysregulation in both SAT-derived chemerin and omentin1 in subjects with nascent MetS, suggesting a possible role of these adipokines in both diabetes and CVD. Future investigations should study different fat depots to determine if our findings in a predominantly Caucasian population on SAT biology dysregulation are relevant to different race groups. Finally, comparing different fat depots in the same population with nascent metabolic syndrome will help decipher their role in adipokine dysregulation, insulin resistance, and inflammation in the pathobiology of this galloping epidemic.

\section{Conflict of Interests}

There are no potential conflict of interests related to this paper and the authors have nothing to disclose.

\section{Acknowledgments}

The study was supported by Grants from the American Diabetes Association and the National Institutes of Health, Grant nos. KL2 RR024144 and UL1 RR024146, respectively. Its contents are solely the responsibility of the authors and do not necessarily represent the official view of the ADA or NIH.

\section{References}

[1] G. M. Reaven, "The insulin resistance syndrome: definition and dietary approaches to treatment," Annual Review of Nutrition, vol. 25, pp. 391-406, 2005.

[2] R. H. Eckel, S. M. Grundy, and P. Z. Zimmet, "The metabolic syndrome," Lancet, vol. 365, no. 9468, pp. 1415-1428, 2005.

[3] S. M. Haffner and H. B. Cassells, "Metabolic syndrome-a new risk factor of coronary heart disease?" Diabetes, Obesity and Metabolism, vol. 5, no. 6, pp. 359-370, 2003.
[4] M. A. Cornier, D. Dabelea, T. L. Hernandez et al., "The metabolic syndrome," Endocrine Reviews, vol. 29, no. 7, pp. 777-822, 2008.

[5] J. I. Cleeman, "Executive summary of the third report of the National Cholesterol Education Program (NCEP) expert panel on detection, evaluation, and treatment of high blood cholesterol in adults (adult treatment panel III)," Journal of the American Medical Association, vol. 285, no. 19, pp. 2486-2497, 2001.

[6] A. Mozumdar and G. Liguori, "Persistent increase of prevalence of metabolic syndrome among U.S. adults: NHANES III to NHANES 1999-2006," Diabetes Care, vol. 34, no. 1, pp. 216-219, 2011.

[7] C. M. Alexander, P. B. Landsman, S. M. Teutsch, and S. M. Haffner, "NCEP-defined metabolic syndrome, diabetes, and prevalence of coronary heart disease among NHANES III participants age 50 years and older," Diabetes, vol. 52, no. 5 , pp. 1210-1214, 2003.

[8] K. G. M. M. Alberti, R. H. Eckel, S. M. Grundy et al., "Harmonizing the metabolic syndrome: a joint interim statement of the international diabetes federation task force on epidemiology and prevention; National heart, lung, and blood institute; American heart association; World heart federation; International atherosclerosis society; And international association for the study of obesity," Circulation, vol. 120, no. 16, pp. 1640-1645, 2009.

[9] C. Schmidt and G. M. Bergstrom, "The metabolic syndrome predicts cardiovascular events: results of a 13-year follow-up in initially healthy 58-year-old men," Metabolic Syndrome and Related Disordoers, vol. 10, no. 6, pp. 394-399, 2012.

[10] H. M. Lakka, D. E. Laaksonen, T. A. Lakka et al., “The metabolic syndrome and total and cardiovascular disease mortality in middle-aged men," Journal of the American Medical Association, vol. 288, no. 21, pp. 2709-2716, 2002.

[11] R. L. Hanson, G. Imperatore, P. H. Bennett, and W. C. Knowler, "Components of the "metabolic syndrome" and incidence of type 2 diabetes," Diabetes, vol. 51, no. 10, pp. 3120-3127, 2002.

[12] G. Assmann, J. R. Nofer, and H. Schulte, "Cardiovascular risk assessment in metabolic syndrome: view from PROCAM," Endocrinology and Metabolism Clinics of North America, vol. 33, no. 2, pp. 377-392, 2004.

[13] S. Devaraj, R.S. Rosenson, and I. Jialal, "Metabolic syndrome: an appraisal of the pro-inflammatory and procoagulant status," Endocrinology and Metabolism Clinics of North America, vol. 33, pp. 431-453, 2004.

[14] S. Devaraj, D. Siegel, and I. Jialal, "Inflammation and metabolic syndrome," in The Metabolic Syndrome, C. D. Byrne and S.H. Wild, Eds., ch 13, pp. 210-228, Wiley-Blackwell, 2nd edition, 2011.

[15] J. M. Northcott, A. Yeganeh, C. G. Taylor, P. Zahradka, and J. T. Wigle, "Adipokines and the cardiovascular system: mechanisms mediating health and disease," Canadian Journal of Physiology and Pharmacology, vol. 90, no. 8, pp. 1029-1059, 2012.

[16] M. Bluher, “Clinical relevance of adipokines," Diabetes and Metabolism Journal, vol. 36, no. 5, pp. 317-327, 2012.

[17] I. Jialal, S. Devaraj, B. A. Huet, X. Chen, and H. Kaur, "Increased cellular and circulating biomarkers of oxidative stress in nascent metabolic syndrome," Journal of Clinical Endocrinology and Metabolism, vol. 97, no. 10, pp. E1844-E1850, 2012.

[18] G. S. Hotamisligil, "Inflammation and metabolic disorders," Nature, vol. 444, no. 7121, pp. 860-867, 2006. 
[19] R. Monteiro and I. Azevedo, "Chronic inflammation in obesity and the metabolic syndrome," Mediators of Inflammation, vol. 2010, Article ID 289645, 10 pages, 2010.

[20] J. P. Despres, S. Lemieux, B. Lamarche et al., "The insulin resistance dyslipidemic syndrome: contribution of visceral obesity and therapeutic implications," International Journal of Obesity, vol. 19, no. 1, supplement, pp. S76-S86, 1995.

[21] J. P. Després, "Is visceral obesity the cause of the metabolic syndrome?" Annals of Medicine, vol. 38, no. 1, pp. 52-63, 2006.

[22] C. S. Fox, J. M. Massaro, U. Hoffmann et al., "Abdominal visceral and subcutaneous adipose tissue compartments: association with metabolic risk factors in the framingham heart study," Circulation, vol. 116, no. 1, pp. 39-48, 2007.

[23] S. A. Porter, J. M. Massaro, U. Hoffmann, R. S. Vasan, C. J. O'Donnel, and C. S. Fox, "Abdominal subcutaneous adipose tissue: a protective fat depot?” Diabetes Care, vol. 32, no. 6, pp. 1068-1075, 2009.

[24] B. H. Goodpaster, F. L. Thaete, J. A. Simoneau, and D. E. Kelley, "Subcutaneous abdominal fat and thigh muscle composition predict insulin sensitivity independently of visceral fat," Diabetes, vol. 46, no. 10, pp. 1579-1585, 1997.

[25] N. Abate, A. Garg, R. M. Peshock, J. Stray-Gundersen, and S. M. Grundy, "Relationships of generalized and regional adiposity to insulin sensitivity in men," Journal of Clinical Investigation, vol. 96, no. 1, pp. 88-98, 1995.

[26] N. Abate, A. Garg, R. M. Peshock, J. Stray-Gundersen, B. Adams-Huet, and S. M. Grundy, "Relationship of generalized and regional adiposity to insulin sensitivity in men with NIDDM," Diabetes, vol. 45, no. 12, pp. 1684-1693, 1996.

[27] I. Ferreira, R. M. A. Henry, J. W. R. Twisk, W. Van Mechelen, H. C. G. Kemper, and C. D. A. Stehouwer, "The metabolic syndrome, cardiopulmonary fitness, and subcutaneous trunk fat as independent determinants of arterial stiffness. The Amsterdam Growth and Health Longitudinal Study," Archives of Internal Medicine, vol. 165, no. 8, pp. 875-882, 2005.

[28] U. Salmenniemi, E. Ruotsalainen, J. Pihlajamäki et al., "Multiple abnormalities in glucose and energy metabolism and coordinated changes in levels of adiponectin, cytokines, and adhesion molecules in subjects with metabolic syndrome," Circulation, vol. 110, no. 25, pp. 3842-3848, 2004.

[29] D. B. Carr, K. M. Utzschneider, R. L. Hull et al., "Intraabdominal fat is a major determinant of the National Cholesterol Education Program Adult Treatment Panel III criteria for the metabolic syndrome," Diabetes, vol. 53, no. 8, pp. 2087-2094, 2004.

[30] C. M. Apovian, S. Bigornia, M. Mott et al., "Adipose macrophage infiltration is associated with insulin resistance and vascular endothelial dysfunction in obese subjects," Arteriosclerosis, Thrombosis, and Vascular Biology, vol. 28, no. 9, pp. 1654-1659, 2008.

[31] A. A. Bremer, S. Devaraj, A. Afify, and I. Jialal, "Adipose tissue dysregulation in patients with metabolic syndrome," Journal of Clinical Endocrinology and Metabolism, vol. 96, no. 11, pp. E1782-E1788, 2011.

[32] S. Devaraj, G. Jialal, T. Cook, D. Siegel, and I. Jialal, "Low vitamin D levels in Northern American adults with the metabolic syndrome," Hormone and Metabolic Research, vol. 43, no. 1, pp. 72-74, 2011.

[33] E. K. Anderson, D. A. Gutierrez, and A. H. Hasty, "Adipose tissue recruitment of leukocytes," Current Opinion in Lipidology, vol. 21, no. 3, pp. 172-177, 2010.
[34] B. K. Surmi and A. H. Hasty, "Macrophage infiltration into adipose tissue: initiation, propagation and remodeling," Future Lipidology, vol. 3, no. 5, pp. 545-556, 2008.

[35] I. S. Wood, F. P. De Heredia, B. Wang, and P. Trayhurn, "Cellular hypoxia and adipose tissue dysfunction in obesity," Proceedings of the Nutrition Society, vol. 68, no. 4, pp. 370-377, 2009.

[36] S. Gormez, A. Demirkan, F. Atalar et al., "Adipose tissue gene expression of adiponectin, tumor necrosis factor- $\alpha$ and leptin in metabolic syndrome patients with coronary artery disease," Internal Medicine, vol. 50, no. 8, pp. 805-810, 2011.

[37] H. S. Sacks, J. N. Fain, P. Cheema et al., "Inflammatory genes in epicardial fat contiguous with coronary atherosclerosis in the metabolic syndrome and type 2 diabetes: changes associated with pioglitazone," Diabetes Care, vol. 34, no. 3, pp. 730-733, 2011.

[38] G. R. Hajer, T. W. Van Haeften, and F. L. J. Visseren, "Adipose tissue dysfunction in obesity, diabetes, and vascular diseases," European Heart Journal, vol. 29, no. 24, pp. 2959-2971, 2008.

[39] G. Fantuzzi and T. Mazzone, "Adipose tissue and atherosclerosis: exploring the connection," Arteriosclerosis, Thrombosis, and Vascular Biology, vol. 27, no. 5, pp. 996-1003, 2007.

[40] Y. Deng and P. E. Scherer, "Adipokines as novel biomarkers and regulators of the metabolic syndrome," Annals of the New York Academy of Sciences, vol. 1212, pp. E1-E19, 2010.

[41] N. Ouchi, S. Kihara, T. Funahashi et al., "Reciprocal association of C-reactive protein with adiponectin in blood stream and adipose tissue," Circulation, vol. 107, no. 5, pp. 671-674, 2003.

[42] S. K. Venugopal, S. Devaraj, and I. Jialal, "Macrophage conditioned medium induces the expression of C-reactive protein in human aortic endothelial cells: potential for paracrine/autocrine effects," American Journal of Pathology, vol. 166, no. 4, pp. 1265-1271, 2005.

[43] I. Huang-Doran, A. Sleigh, J. J. Rochford, S. O’Rahilly, and D. B. Savage, "Lipodystrophy: metabolic insights from a rare disorder," Journal of Endocrinology, vol. 207, no. 3, pp. 245-255, 2010.

[44] C. Mitchell, D. Couton, J. P. Couty et al., "Dual role of CCR2 in the constitution and the resolution of liver fibrosis in mice," American Journal of Pathology, vol. 174, no. 5, pp. 1766-1775, 2009.

[45] A. Ito, T. Suganami, A. Yamauchi et al., "Role of CC chemokine receptor 2 in bone marrow cells in the recruitment of macrophages into obese adipose tissue," Journal of Biological Chemistry, vol. 283, no. 51, pp. 35715-35723, 2008.

[46] M. C. Ernst and C. J. Sinal, "Chemerin: at the crossroads of inflammation and obesity," Trends in Endocrinology and Metabolism, vol. 21, no. 11, pp. 660-667, 2010.

[47] K. Bozaoglu, D. Segal, K. A. Shields et al., "Chemerin is associated with metabolic syndrome phenotypes in a MexicanAmerican population," Journal of Clinical Endocrinology and Metabolism, vol. 94, no. 8, pp. 3085-3088, 2009.

[48] H. Sell, A. Divoux, C. Poitou et al., "Chemerin correlates with markers for fatty liver in morbidly obese patients and strongly decreases after weight loss induced by bariatric surgery," Journal of Clinical Endocrinology and Metabolism, vol. 95, no. 6, pp. 2892-2896, 2010.

[49] H. Sell, J. Laurencikiene, A. Taube et al., "Chemerin is a novel adipocyte-derived factor inducing insulin resistance in primary human skeletal muscle cells," Diabetes, vol. 58, no. 12, pp. 27312740, 2009. 
[50] D. Stejskal, M. Karpisek, Z. Hanulova, and M. Svestak, "Chemerin is an independent marker of the metabolic syndrome in a Caucasian population-a pilot study," Biomedical Papers of the Medical Faculty of the University Palacký, Olomouc, Czechoslovakia, vol. 152, no. 2, pp. 217-221, 2008.

[51] S. H. Chu, M. K. Lee, K. Y. Ahn et al., "Chemerin and adiponectin contribute reciprocally to metabolic syndrome," PLoS ONE, vol. 7, no. 4, Article ID e34710, 2012.

[52] B. Dong, W. Ji, and Y. Zhang, "Elevated serum chemerin levels are associated with the Presence of coronary artery disease in patients with metabolic syndrome," Internal Medicine, vol. 50, no. 10, pp. 1093-1097, 2011.

[53] J. Weigert, M. Neumeier, J. Wanninger et al., "Systemic chemerin is related to inflammation rather than obesity in type 2 diabetes," Clinical Endocrinology, vol. 72, no. 3, pp. 342-348, 2010.

[54] A. Schäffler, M. Neumeier, H. Herfarth, A. Fürst, J. Schölmerich, and C. Büchler, "Genomic structure of human omentin, a new adipocytokine expressed in omental adipose tissue," Biochimica et Biophysica Acta, vol. 1732, no. 1-3, pp. 96-102, 2005.

[55] B. K. Tan, R. Adya, and H. S. Randeva, "Omentin: a novel link between inflammation, diabesity, and cardiovascular disease," Trends in Cardiovascular Medicine, vol. 20, no. 5, pp. 143-148, 2010.

[56] C. M. De Souza Batista, R. Z. Yang, M. J. Lee et al., "Omentin plasma levels and gene expression are decreased in obesity," Diabetes, vol. 56, no. 6, pp. 1655-1661, 2007.

[57] F. J. Shang, J. P. Wang, X. T. Liu et al., "Serum omentin-1 levels are inversely associated with the presence and severity of coronary artery disease in patients with metabolic syndrome," Biomarkers, vol. 16, no. 8, pp. 657-662, 2011.

[58] E. Gremese and G. Ferraccioli, "The metabolic syndrome: the crossroads between rheumatoid arthritis and cardiovascular risk," Autoimmunity Reviews, vol. 10, no. 10, pp. 582-589, 2011.

[59] I. Jialal, S. Devaraj, H. Kaur, B. A. Huet, and A. A. Bremer, "Increased chemerin and decreased omentin-1 in both adipose tissue and plasma in nascent metabolic syndrome," Journal of Clinical Endocrinology and Metabolism, 2013.

[60] I. Jialal, B. A. Huet, H. Kaur, A. Chien, and S. Devaraj, "Increased toll-like receptor activity in patients with metabolic syndrome," Diabetes Care, vol. 35, no. 4, pp. 900-904, 2012. 


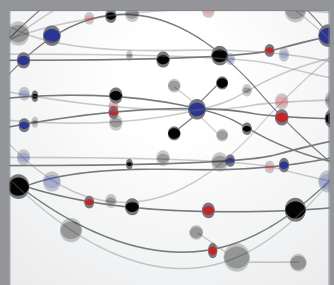

The Scientific World Journal
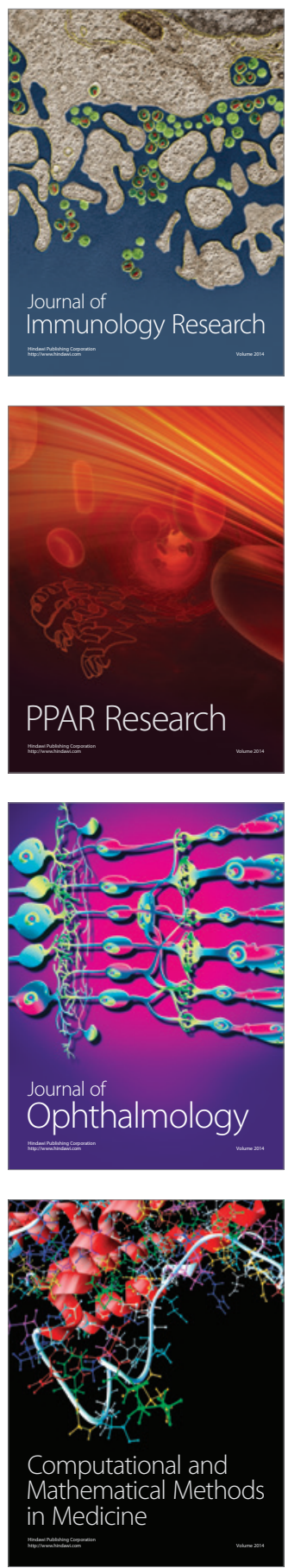

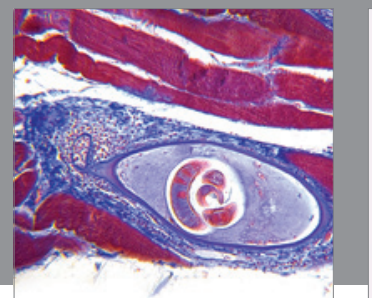

Gastroenterology

Research and Practice
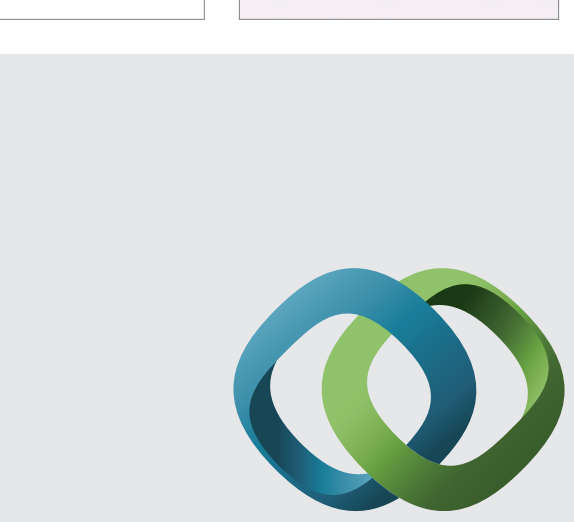

\section{Hindawi}

Submit your manuscripts at

http://www.hindawi.com
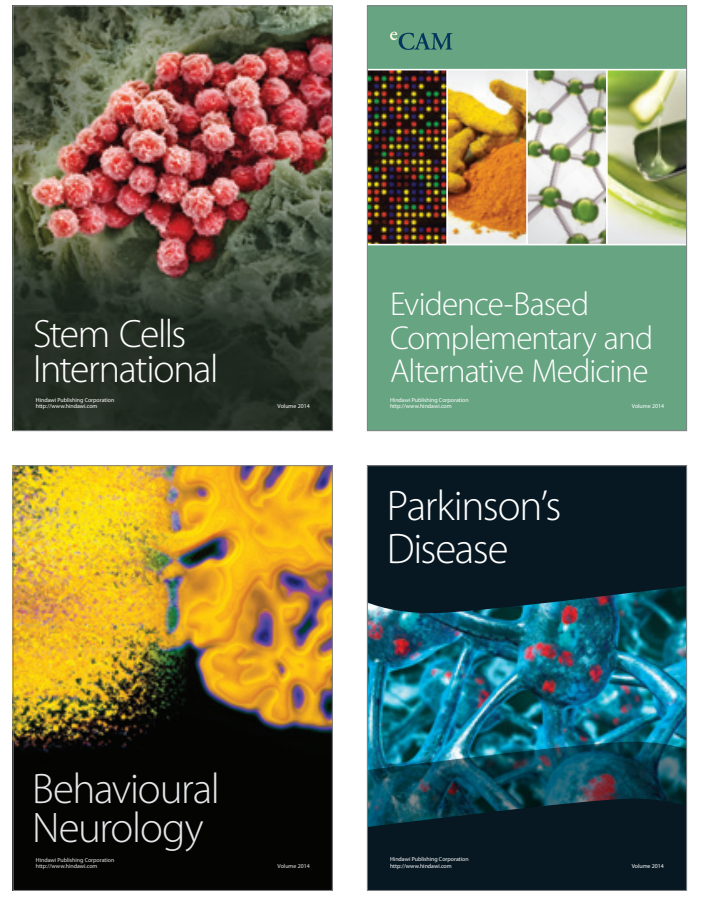
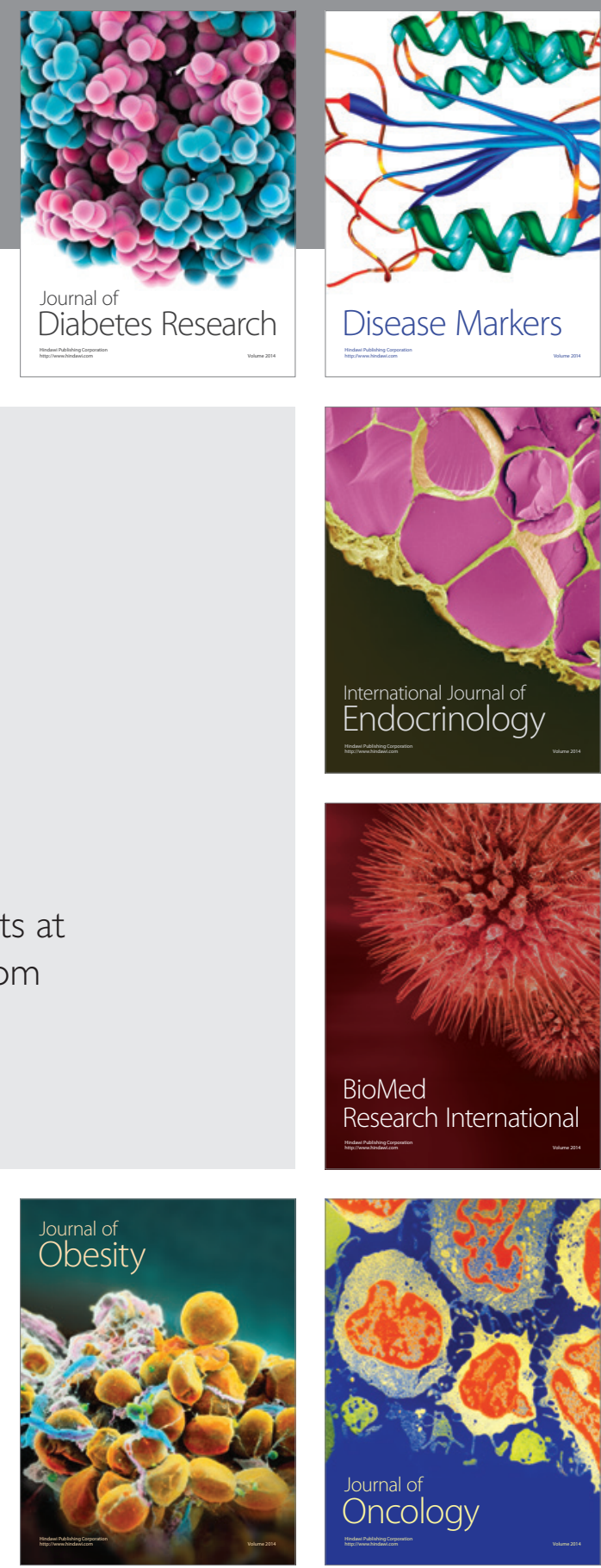

Disease Markers
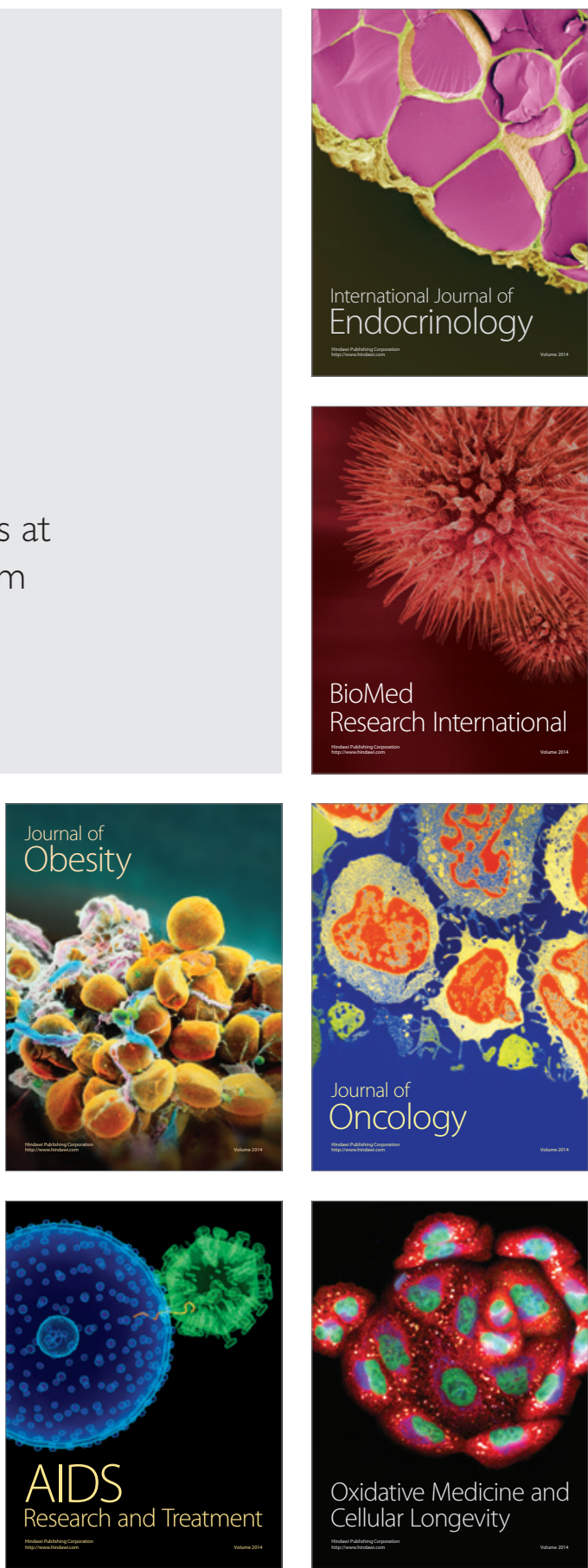